\title{
Krasnoselskii-Mann method for non-self mappings
}

\author{
Vittorio Colao ${ }^{1}$ and Giuseppe Marino ${ }^{1,2^{*}}$
}

${ }^{\text {*Correspondence: }}$ giuseppe.marino@unical.it

${ }^{1}$ Department of Mathematics and Computer Science, Universitá della Calabria, Rende, CS, Italy ${ }^{2}$ Department of Mathematics, King Abdulaziz University, P.O. Box 80203, Jeddah, 21589, Saudi Arabia

\begin{abstract}
Let $H$ be a Hilbert space and let $C$ be a closed, convex and nonempty subset of $H$. If $T: C \rightarrow H$ is a non-self and non-expansive mapping, we can define a map $h: C \rightarrow \mathbb{R}$ by $h(x):=\inf \{\lambda \geq 0: \lambda x+(1-\lambda) T x \in C\}$. Then, for a fixed $x_{0} \in C$ and for $\alpha_{0}:=\max \{1 / 2$, $\left.h\left(x_{0}\right)\right\}$, we define the Krasnoselskii-Mann algorithm $x_{n+1}=\alpha_{n} x_{n}+\left(1-\alpha_{n}\right) T x_{n}$, where $\alpha_{n+1}=\max \left\{\alpha_{n}, h\left(x_{n+1}\right)\right\}$. We will prove both weak and strong convergence results when $C$ is a strictly convex set and $T$ is an inward mapping.
\end{abstract}

\section{Introduction}

Let $C$ be a closed, convex and nonempty subset of a Hilbert space $H$ and let $T: C \rightarrow H$ be a non-expansive mapping such that the fixed point set $\operatorname{Fix}(T):=\{x \in C: T x=x\}$ is not empty.

For a real sequence $\left\{\alpha_{n}\right\} \subset(0,1)$, we will consider the iterations

$$
\left\{\begin{array}{l}
x_{0} \in C \\
x_{n+1}=\alpha_{n} x_{n}+\left(1-\alpha_{n}\right) T x_{n} .
\end{array}\right.
$$

If $T$ is a self-mapping, the iterative scheme above has been studied in an impressive amount of papers (see [1] and the references therein) in the last decades and it is often called 'segmenting Mann' [2-4] or 'Krasnoselskii-Mann' (e.g., [5, 6]) iteration.

A general result on algorithm (1) is due to Reich [7] and states that the sequence $\left\{x_{n}\right\}$ weakly converges to a fixed point of the operator $T$ under the following assumptions:

(C1) $T$ is a self-mapping, i.e., $T: C \rightarrow C$ and

(C2) $\left\{\alpha_{n}\right\}$ is such that $\sum_{n} \alpha_{n}\left(1-\alpha_{n}\right)=+\infty$.

In this paper, we are interested in lowering condition (C1) by allowing $T$ to be non-self at the price of strengthening the requirements on the sequence $\left\{\alpha_{n}\right\}$ and on the set $C$. Indeed, we will assume that $C$ is a strictly convex set and that the non-expansive map $T: C \rightarrow H$ is inward.

Historically, the inward condition and its generalizations were widely used to prove convergence results for both implicit [8-11] and explicit (see, e.g., [1,12-14]) algorithms. However, we point out that the explicit case was only studied in conjunction with processes involving the calculation of a projection or a retraction $P: H \rightarrow C$ at each step.

As an example, in [12], the following algorithm is studied:

$$
x_{n+1}=P\left(\alpha_{n} f\left(x_{n}\right)+\left(1-\alpha_{n}\right) T x_{n}\right) \text {, }
$$

( 2015 Colao and Marino; licensee Springer. This is an Open Access article distributed under the terms of the Creative Commons Attribution License (http://creativecommons.org/licenses/by/4.0), which permits unrestricted use, distribution, and reproduction in any medium, provided the original work is properly credited. 
where $T: C \rightarrow H$ satisfies the weakly inward condition, $f$ is a contraction and $P: H \rightarrow C$ is a non-expansive retraction.

We point out that in many real world applications, the process of calculating $P$ can be a resource consumption task and it may require an approximating algorithm by itself, even in the case when $P$ is the nearest point projection.

To overcome the necessity of using an auxiliary mapping $P$, for an inward and nonexpansive mapping $T: C \rightarrow H$, we will introduce a new search strategy for the coefficients $\left\{\alpha_{n}\right\}$ and we will prove that the Krasnoselskii-Mann algorithm

$$
x_{n+1}=\alpha_{n} x_{n}+\left(1-\alpha_{n}\right) T x_{n}
$$

is well defined for this particular choice of the sequence $\left\{\alpha_{n}\right\}$. Also we will prove both weak and strong convergence results for the above algorithm when $C$ is a strictly convex set.

We stress that the main difference between the classical Krasnoselskii-Mann and our algorithm is that the choice of the coefficient $\alpha_{n}$ is not made a priori in the latter, but it is constructed step to step and determined by the values of the map $T$ and the geometry of the set $C$.

\section{Main result}

We will make use of the following.

Definition 1 A map $T: C \rightarrow H$ is said to be inward (or to satisfy the inward condition) if, for any $x \in C$, it holds

$$
T x \in I_{C}(x):=\{x+c(u-x): c \geq 1 \text { and } u \in C\} .
$$

We refer to [15] for a comprehensive survey on the properties of the inward mappings.

Definition 2 A set $C \subset H$ is said to be strictly convex if it is convex and with the property that $x, y \in \partial C$ and $t \in(0,1)$ implies that

$$
t x+(1-t) y \in \stackrel{\circ}{C} .
$$

In other words, if the boundary $\partial C$ does not contain any segment.

Definition 3 A sequence $\left\{y_{n}\right\} \subset C$ is Fejér-monotone with respect to a set $D \subset C$ if, for any element $y \in D$,

$$
\left\|y_{n+1}-y\right\| \leq\left\|y_{n}-y\right\| \quad \forall n \in \mathbb{N} .
$$

For a closed and convex set $C$ and a map $T: C \rightarrow H$, we define a mapping $h: C \rightarrow \mathbb{R}$ as

$$
h(x):=\inf \{\lambda \geq 0: \lambda x+(1-\lambda) T x \in C\} .
$$

Note that the above quantity is a minimum since $C$ is closed. In the following lemma, we group the properties of the function defined above. 
Lemma 1 Let $C$ be a nonempty, closed and convex set, let $T: C \rightarrow H$ be a mapping and define $h: C \rightarrow \mathbb{R}$ as in (3). Then the following properties hold:

(P1) for any $x \in C, h(x) \in[0,1]$ and $h(x)=0$ if and only if $T x \in C$;

(P2) for any $x \in C$ and any $\alpha \in[h(x), 1], \alpha x+(1-\alpha) T x \in C$;

(P3) if $T$ is an inward mapping, then $h(x)<1$ for any $x \in C$;

(P4) whenever $T x \notin C, h(x) x+(1-h(x)) T x \in \partial C$.

Proof Properties (P1) and (P2) follow directly from the definition of $h$. To prove (P3), observe that (2) implies

$$
\frac{1}{c} T x+\left(1-\frac{1}{c}\right) x \in C
$$

for some $c \geq 1$. As a consequence,

$$
h(x)=\inf \{\lambda \geq 0: \lambda x+(1-\lambda) T x \in C\} \leq\left(1-\frac{1}{c}\right)<1 .
$$

In order to verify (P4), we first note that $h(x)>0$ by property (P1) and that $h(x) x+(1-$ $h(x)) T x \in C$. Let $\left\{\eta_{n}\right\} \subset(0, h(x))$ be a sequence of real numbers converging to $h(x)$ and note that, by the definition of $h$, it holds

$$
z_{n}:=\eta_{n} x+\left(1-\eta_{n}\right) T x \notin C
$$

for any $n \in \mathbb{N}$. Since $\eta_{n} \rightarrow h(x)$ and

$$
\left\|z_{n}-h(x) x-(1-h(x)) T x\right\|=\left|\eta_{n}-h(x)\right|\|x-T x\|,
$$

it follows that $z_{n} \rightarrow h(x) x+(1-h(x)) T x \in C$, so that this last must belong to $\partial C$.

Our main result is the following.

Theorem 1 Let $C$ be a convex, closed and nonempty subset of a Hilbert space $H$ and let $T: C \rightarrow H$ be a mapping. Then the algorithm

$$
\left\{\begin{array}{l}
x_{0} \in C, \\
\alpha_{0}:=\max \left\{\frac{1}{2}, h\left(x_{0}\right)\right\} \\
x_{n+1}:=\alpha_{n} x_{n}+\left(1-\alpha_{n}\right) T x_{n}, \\
\alpha_{n+1}:=\max \left\{\alpha_{n}, h\left(x_{n+1}\right)\right\}
\end{array}\right.
$$

is well defined.

If we further assume that

1. $C$ is strictly convex and

2. $T$ is a non-expansive mapping, which satisfies the inward condition (2) and such that $\operatorname{Fix}(T) \neq \emptyset$,

then $\left\{x_{n}\right\}$ weakly converges to a point $p \in \operatorname{Fix}(T)$. Moreover, if $\sum_{n=0}^{\infty}\left(1-\alpha_{n}\right)<\infty$, then the convergence is strong. 
Proof To prove that the algorithm is well defined, it is sufficient to note that $\alpha_{n} \in\left[h\left(x_{n}\right), 1\right]$ for any $n \in \mathbb{N}$; then, by recalling property (P2) from Lemma 1, it immediately follows that

$$
x_{n+1}=\alpha_{n} x_{n}+\left(1-\alpha_{n}\right) T x_{n} \in C .
$$

Assume now that $T$ satisfies the inward condition. In this case, by property (P3) of the previous lemma, we obtain that the non-decreasing sequence $\left\{\alpha_{n}\right\}$ is contained in $\left[\frac{1}{2}, 1\right)$. Also, since $T$ is non-expansive and with at least one fixed point, it follows by standard arguments that $\left\{x_{n}\right\}$ is Fejér-monotone with respect to Fix $(T)$ and, as a consequence, both $\left\{x_{n}\right\}$ and $\left\{T x_{n}\right\}$ are bounded.

Firstly, assume that $\sum_{n=0}^{\infty}\left(1-\alpha_{n}\right)=\infty$. Then, since $\alpha_{n} \geq \frac{1}{2}$, we derive that $\sum_{n=0}^{\infty} \alpha_{n}(1-$ $\left.\alpha_{n}\right)=\infty$ and from Lemma 2 of [16] we obtain that

$$
\left\|x_{n}-T x_{n}\right\| \rightarrow 0
$$

This fact, together with the Fejér-monotonicity of $\left\{x_{n}\right\}$ proves that the sequence weakly converges in Fix $(T)$ (see [17], Proposition 2.1).

Suppose that

$$
\sum_{n=0}^{\infty}\left(1-\alpha_{n}\right)<\infty .
$$

Since

$$
\left\|x_{n+1}-x_{n}\right\|=\left(1-\alpha_{n}\right)\left\|T x_{n}-x_{n}\right\|,
$$

and by the boundedness of $\left\{x_{n}\right\}$ and $\left\{T x_{n}\right\}$, it is promptly obtained that

$$
\sum_{n=0}^{\infty}\left\|x_{n+1}-x_{n}\right\|<\infty \text {, }
$$

i.e., $\left\{x_{n}\right\}$ is a strongly Cauchy sequence and hence $x_{n} \rightarrow x^{*} \in C$.

Note that $T$ satisfies the inward condition. Then, by applying properties (P2) and (P3) from Lemma 1, we obtain that $h\left(x^{*}\right)<1$ and that for any $\mu \in\left(h\left(x^{*}\right), 1\right)$ it holds

$$
\mu x^{*}+(1-\mu) T x^{*} \in C .
$$

On the other hand, we observe that since $\lim _{n \rightarrow \infty} \alpha_{n}=1$ by (5) and since $\alpha_{n}=\max \left\{\alpha_{n-1}\right.$, $\left.h\left(x_{n}\right)\right\}$ holds, it follows that we can choose a sub-sequence $\left\{x_{n_{k}}\right\}$ with the property that $\left\{h\left(x_{n_{k}}\right)\right\}$ is non-decreasing and $h\left(x_{n_{k}}\right) \rightarrow 1$. In particular, for any $\mu<1$,

$$
\mu x_{n_{k}}+(1-\mu) T x_{n_{k}} \notin C
$$

eventually holds.

Choose $\mu_{1}, \mu_{2} \in\left(h\left(x^{*}\right), 1\right)$ with $\mu_{1} \neq \mu_{2}$ and set $v_{1}:=\mu_{1} x^{*}+\left(1-\mu_{1}\right) T x^{*}$ and $v_{2}:=\mu_{2} x^{*}+$ $\left(1-\mu_{2}\right) T x^{*}$. Then, whenever $\mu \in\left[\mu_{1}, \mu_{2}\right]$, by (6) we have that $v:=\mu x^{*}+(1-\mu) T x^{*} \in C$. 
Moreover,

$$
\mu x_{n_{k}}+(1-\mu) T x_{n_{k}} \rightarrow v
$$

since $x_{n} \rightarrow x^{*}$. This last, together with (7), implies that $v \in \partial C$ and $\left[v_{1}, v_{2}\right] \subset \partial C$, since $\mu$ is arbitrary.

By the strict convexity of $C$, we derive that

$$
\mu_{1} x^{*}+\left(1-\mu_{1}\right) T x^{*}=\mu_{2} x^{*}+\left(1-\mu_{2}\right) T x^{*}
$$

and $x^{*}=T x^{*}$ must necessarily hold, i.e., $\left\{x_{n}\right\}$ strongly converges to a fixed point of $T$.

Remark 1 Following the same line of proof, it can be easily seen that the same results hold true if the starting coefficient $\alpha_{0}=\max \left\{\frac{1}{2}, h\left(x_{0}\right)\right\}$ is substituted by $\alpha_{0}=\max \left\{b, h\left(x_{0}\right)\right\}$, where $b \in(0,1)$ is a fixed and arbitrary value. In the statement of Theorem 1 , the value $b=\frac{1}{2}$ was taken to ease the notation.

We also note that the value $h\left(x_{n}\right)$ can be replaced, in practice, by $h_{n}=1-\frac{1}{2^{j} n}$, where $j_{n}:=\min \left\{j \in \mathbb{N}:\left(1-\frac{1}{2^{j}}\right) x_{n}+\frac{1}{2^{j}} T x_{n} \in C\right\}$.

Remark 2 As it follows from the proof, the condition $\sum_{n}\left(1-\alpha_{n}\right)<\infty$ provides a localization result for the fixed point $x^{*}$ as a side result. Indeed, in this case, it holds that $x^{*}=v_{1}=v_{2}$ belongs to the boundary $\partial C$ of the set $C$.

Remark 3 In [18], for a closed and convex set $C$, the map

$$
f(x):=\inf \{\lambda \in[0,1]: x \in \lambda C\}
$$

was introduced and used in conjunction with an iterative scheme to approximate a fixed point of minimum norm (see also [19]). Indeed, in the above mentioned paper, it is proved that the iterative scheme

$$
\left\{\begin{array}{l}
\lambda_{n}=\max \left\{f\left(x_{n}\right), \lambda_{n-1}\right\}, \\
y_{n}=\alpha_{n} x_{n}+\left(1-\alpha_{n}\right) T x_{n}, \\
x_{n+1}=\alpha_{n} \lambda_{n} x_{n}+\left(1-\alpha_{n}\right) y_{n}
\end{array}\right.
$$

strongly converges under the assumptions that $\left\{\alpha_{n}\right\}$ is a sequence in $(0,1)$ such that $\lim _{n} \frac{\alpha_{n}}{\left(1-\lambda_{n}\right)}=0$ and that $\sum_{n}\left(1-\lambda_{n}\right) \alpha_{n}=\infty$. We point out that the mentioned conditions appear to be difficult to be checked as they involve the geometry of the set $C$.

We illustrate the statement of our results with a brief example.

Example 1 Let $H=l^{2}(\mathbb{R})$ and let $C:=B_{1} \cap B_{2}$, where $B_{1}:=\left\{\left(t_{i}\right)_{i \in \mathbb{N}}:\left(t_{1}-49.995\right)^{2}+\right.$ $\left.\sum_{i=2}^{\infty} t_{i}^{2} \leq(50.005)^{2}\right\}$ and $B_{2}:=\left\{\left(t_{i}\right)_{i \in \mathbb{N}}: \sum_{i=1}^{\infty} t_{i}^{2} \leq 1\right\}$. Then $C$ is a nonempty, closed and strictly convex subset of $H$. Let $T: C \rightarrow H$ be the map defined by $T\left(t_{1}, t_{2}, \ldots, t_{i}, \ldots\right):=$ $\left(-t_{1}, t_{2}, \ldots, t_{i}, \ldots\right)$, then $T$ is a non-expansive inward map with $\operatorname{Fix}(T)=\left\{\left(0, t_{2}, \ldots, t_{i}, \ldots\right)\right.$ : 
$\left.\sum_{i=2}^{\infty} t_{i}^{2} \leq 1\right\}$. If we use the algorithm

$$
\left\{\begin{array}{l}
x_{0}=\left(t_{i}\right)_{i \in \mathbb{N}} \in C, \\
\alpha_{0}:=\max \left\{\frac{1}{2}, h\left(x_{0}\right)\right\}, \\
x_{n+1}:=\alpha_{n} x_{n}+\left(1-\alpha_{n}\right) T x_{n}, \\
\alpha_{n+1}:=\max \left\{\alpha_{n}, h\left(x_{n+1}\right)\right\},
\end{array}\right.
$$

then, by the natural symmetry of the problem, we obtain the constant sequence

$$
x_{1}=\cdots=x_{n}=\left(0, t_{2}, \ldots, t_{i}, \ldots\right) \in \operatorname{Fix}(T) .
$$

If we use the algorithm

$$
\left\{\begin{array}{l}
x_{0}=\left(t_{i}\right)_{i \in \mathbb{N}} \in C, \\
\alpha_{0}:=\max \left\{0.01, h\left(x_{0}\right)\right\}, \\
x_{n+1}:=\alpha_{n} x_{n}+\left(1-\alpha_{n}\right) T x_{n}, \\
\alpha_{n+1}:=\max \left\{\alpha_{n}, h\left(x_{n+1}\right)\right\},
\end{array}\right.
$$

then $\left\{x_{n}\right\}$ still converges in $\operatorname{Fix}(T)$, but $\left\{x_{n}\right\} \cap \operatorname{Fix}(T)=\emptyset$ whenever $t_{i} \neq 0$.

We conclude the paper by including few question that appear to be still open to the best of our knowledge.

Question 1 It has been proved that the Krasnoselskii-Mann algorithm converges for general classes of mappings (see, e.g., [20] and [21]). By maintaining the same assumption on the set $C$ and the inward condition of the involved map, it appears to be natural to ask for which classes of mappings the same result of Theorem 1 still holds.

Question 2 Under which assumptions can algorithm (4) be adapted to produce a converging sequence to a common fixed point for a family of mappings? In other words, does the algorithm

$$
\left\{\begin{array}{l}
x_{0} \in C, \\
\alpha_{0}:=\max \left\{\frac{1}{2}, h_{n}\left(x_{0}\right)\right\}, \\
x_{n+1}:=\alpha_{n} x_{n}+\left(1-\alpha_{n}\right) T_{n} x_{n}, \\
\alpha_{n+1}:=\max \left\{\alpha_{n}, h_{n+1}\left(x_{n+1}\right)\right\}
\end{array}\right.
$$

converge to a common fixed point of the family $\left\{T_{n}\right\}$, where

$$
h_{n}(x):=\inf \left\{\lambda \geq 0: \lambda x+(1-\lambda) T_{n} x \in C\right\}
$$

and under suitable hypotheses?

We refer to [22] and [23] for two examples regarding the classical Krasnoselskii-Mann algorithm. 
Question 3 In the classical literature, it has been proved that the inward condition can be often dropped in favor of a weaker condition. For example, a mapping $T: C \rightarrow X$ is said to be weakly inward (or to satisfy the weakly inward condition) if

$$
T x \in \overline{I_{C}(x)} \quad \forall x \in C
$$

Does Theorem 1 hold even for weakly inward mappings?

On the other hand, we observe that the strict convexity of the set $C$ does appear to be unusual for results regarding the convergence of Krasnoselskii-Mann iterations. We do not know if our result can hold for a convex and $\operatorname{closed} \operatorname{set} C$, even at the price of strengthening the requirements on the map $T$.

\section{Competing interests}

The authors declare that they have no competing interests.

\section{Authors' contributions}

All authors contributed equally and significantly in writing the article. All authors read and approved the final manuscript.

\section{Acknowledgements}

This project was funded by Ministero dell'Istruzione, dell'Universitá e della Ricerca (MIUR).

Received: 4 December 2014 Accepted: 3 March 2015 Published online: 13 March 2015

\section{References}

1. Chidume, C: Geometric Properties of Banach Spaces and Nonlinear Iterations. Lecture Notes in Mathematics, vol. 1965. Springer, Berlin (2009)

2. Mann, WR: Mean value methods in iteration. Proc. Am. Math. Soc. 4(3), 506-510 (1953)

3. Groetsch, CW: A note on segmenting Mann iterates. J. Math. Anal. Appl. 40(2), 369-372 (1972)

4. Hicks, TL, Kubicek, JD: On the Mann iteration process in a Hilbert space. J. Math. Anal. Appl. 59(3), 498-504 (1977)

5. Edelstein, M, O'Brien, RC: Nonexpansive mappings, asymptotic regularity and successive approximations. J. Lond. Math. Soc. 2(3), 547-554 (1978)

6. Hillam, BP: A generalization of Krasnoselski's theorem on the real line. Math. Mag. 48(3), 167-168 (1975)

7. Reich, S: Weak convergence theorems for nonexpansive mappings in Banach spaces. J. Math. Anal. Appl. 67(2), 274-276 (1979)

8. Xu, H-K, Yin, X-M: Strong convergence theorems for nonexpansive nonself-mappings. Nonlinear Anal. 24(2), 223-228 (1995)

9. Xu, H-K: Approximating curves of nonexpansive nonself-mappings in Banach spaces. C. R. Acad. Sci. Paris Sér. I Math. 325(2), 151-156 (1997)

10. Marino, G, Trombetta, G: On approximating fixed points for nonexpansive mappings. Indian J. Math. 34, 91-98 (1992)

11. Takahashi, W, Kim, G-E: Strong convergence of approximants to fixed points of nonexpansive nonself-mappings in Banach spaces. Nonlinear Anal. 32(3), 447-454 (1998)

12. Song, Y, Chen, R: Viscosity approximation methods for nonexpansive nonself-mappings. J. Math. Anal. Appl. 321(1), 316-326 (2006)

13. Song, YS, Cho, YJ: Averaged iterates for non-expansive nonself mappings in Banach spaces. J. Comput. Anal. Appl. 11, 451-460 (2009)

14. Zhou, H, Wang, P: Viscosity approximation methods for nonexpansive nonself-mappings without boundary conditions. Fixed Point Theory Appl. 2014, 61 (2014)

15. Kirk, W, Sims, B: Handbook of Metric Fixed Point Theory. Springer, Berlin (2001)

16. Ishikawa, S: Fixed points and iteration of a nonexpansive mapping in a Banach space. Proc. Am. Math. Soc. 59(1), 65-71 (1976)

17. Bauschke, $\mathrm{HH}$, Combettes, PL: A weak-to-strong convergence principle for Fejér-monotone methods in Hilbert spaces. Math. Oper. Res. 26(2), 248-264 (2001)

18. He, S, Zhu, W: A modified Mann iteration by boundary point method for finding minimum-norm fixed point of nonexpansive mappings. Abstr. Appl. Anal. 2013, Article ID 768595 (2013)

19. He, S, Yang, C: Boundary point algorithms for minimum norm fixed points of nonexpansive mappings. Fixed Point Theory Appl. 2014, 56 (2014)

20. Schu, J: Iterative construction of fixed points of asymptotically nonexpansive mappings. J. Math. Anal. Appl. 158(2), 407-413 (1991)

21. Marino, G, Xu, H-K: Weak and strong convergence theorems for strict pseudo-contractions in Hilbert spaces. J. Math. Anal. Appl. 329(1), 336-346 (2007)

22. Bauschke, $\mathrm{HH}$ : The approximation of fixed points of compositions of nonexpansive mappings in Hilbert space. J. Math. Anal. Appl. 202(1), 150-159 (1996)

23. Suzuki, T: Strong convergence of Krasnoselskii and Mann's type sequences for one-parameter nonexpansive semigroups without Bochner integrals. J. Math. Anal. Appl. 305(1), 227-239 (2005) 